\title{
Does an internet gaming disorder prospectively predict psychiatric symptoms?
}

By Dr. Jessica Edwards

A minority of children and adolescents develop addiction-like engagement in gaming that is associated with impaired function. ${ }^{1}$ Preliminary data suggest that affected children with these symptoms, indicating an Internet Gaming Disorder (IGD), might present with more symptoms of common psychiatric disorders than those without an IGD. ${ }^{2}$ However, the reasons underlying this co-occurrence are unclear. Now, researchers in Norway have examined the links between the symptoms of an IGD and of common psychiatric disorders to determine whether the observed comorbidity is due to a reciprocal relationship or a common underlying cause.

Beate Wold Hygen and colleagues studied a community sample of 702 Norwegian children who were interviewed to assess for DSM-5defined IGD symptoms at ages 10, 12 and 14 years. They also completed an interview to evaluate symptoms of common psychiatric disorders at the same time points. To assess concurrent and prospective links between symptoms of IGD and other psychopathology, the researchers implemented a random intercept cross-lagged panel model, which adjusts for unmeasured individual factors. Interestingly, they found no associations between IGD symptoms and psychopathology, with the exception that high IGD symptoms at age 10 and 12 years predicted decreased anxiety symptoms 2 years later.

These findings do not support a concurrent or prospective relationship between an IGD and psychiatric symptoms, except for decreased anxiety. Therefore, it seems that the observed co-occurrence between IGD symptoms and psychiatric disorders is attributable to a common aetiology. The researchers hypothesize that genetics may be an important factor, but further work is needed to confirm this hypothesis and to delineate the consistently observed association between extensive gaming and mental health. For now, the researchers propose that heavy gaming during childhood to the extent of developing an IGD might not Referring to:

Wold Hygen, B., Skalická, V., Stenseng, F., Belsky, J., Steinsbekk, S. \& Wichstrøm, L. (2020),

The co-occurrence between symptoms of internet gaming disorder and psychiatric disorders in childhood and adolescence: prospective relations or common causes? J. Child Psychol.

Psychiatr. doi: 10.1111/jcpp.13289.

\section{References:}

${ }^{1}$ Kuss, D.J. et al. (2012), Internet gaming addiction: A systematic review of empirical research. Int.

J. Ment. Health Addiction. 10, 278-296. doi: 10.1007/s11469o11-9318-5.

2 Pearcy, B.T. et al. (2017), Internet gaming disorder explains unique variance in psychological distress and disability after controlling for comorbid depression, $O C D, A D H D$, and anxiety. Cyberpsychol. Behav. Soc. Netw. 20, 126-132. doi: 10.1089/ cyber.2016.0304. confer a risk of developing other mental health problems later in life. 